Mansoura Engineering Journal, (MEJ), Vol.20, No.4. December. 1995. E.61

\title{
FEATURE SELECTION FOR SPEAKER IDENTIFICATION AND ARABIC DIGITS RECOGNITION
}

\section{I. A. ABDALLA* and A, E ELMALLAWANY**}

* Lect, Faculty of Eng. Zagagig Univ.

* Prof. Of Acoustic Eng. Building Research Center.

$$
\text { اختبار خصانت الصصوت للثعرف على المتحدت ور التعرو على ا رقام العربية }
$$

$$
\text { اللحتص العربى }
$$

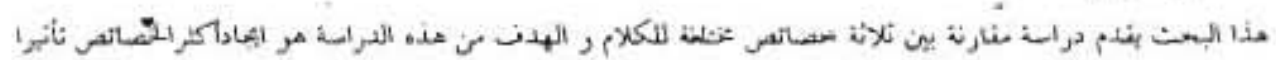

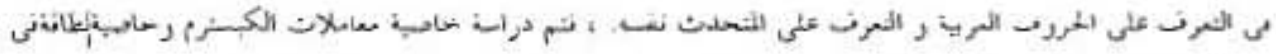

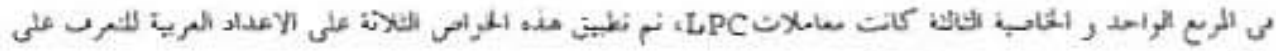

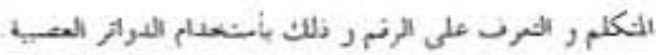

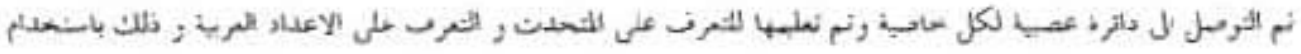

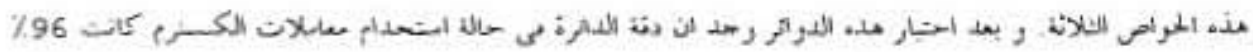

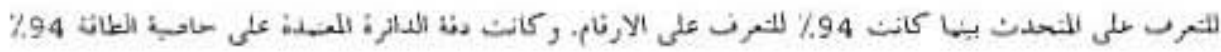

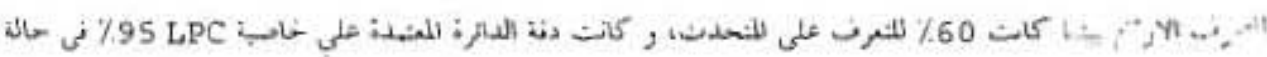

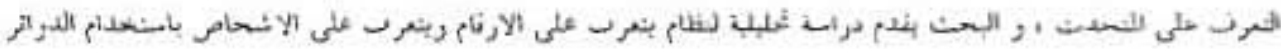

\section{ABSTRACT}

This article introduces a comparison between three different processing techniques for the selection of speech features. These features can be used for speaker recognition or speech recognition. A comparison between the performance of a system based on the linear prediction code, a system based on the cepstrum and a system based on the short time energy is introduced. Feacure selection is very effective for recognition accuracy. This work illustrates where each of chese feacures are more efficient for speaker recognition or for speech recognition.

The results show that the short time energy in time domain is very effective for speech recognition where its accuracy is found to be $92 \%$. In speaker identification, the accuracy of identification for the features depending on energy in each frame is found to be $60 \%$. It may be recommended that the features based on the energy per frame may be used for speech recognition. The features based on the Cepstrum give accuracy of $94 \%$ and $96 \%$ for speech recognition and speaker identification respectively. The accuracy of linear prediction code feacure is found to be $95 \%$ for speaker identification.So the features depend on cepstrum may be recommended for speaker identifier or speech recognition. 
A recognition system for spoken digits are given using the above features with neural networks. The neural network has been used as a tool in this comparison.

1. INTRODUCTION

All the application areas of speech technology, including speech recognition. speech synthesis, speech coding and speaker recognition require some form of preliminary analysis of the speech. Speech analysis techniques may be broadly classified as eicher frequency domain or time domain approaches.

The major goal in speech analysis is to estimate the frequency response of che vocal tract. The techniques of processing the speech signal using a multiple bandpass filter, discrete Fourier cransformation (DFT) and cepstrum can be used to achieve this goal. Time domain measures such as the auco correlation function, zero -crossing rate, and signal energy can also be used to extract useful information about the speech signal. The parameters considered by Walf(1) were pitch at selected point in words, spectral characteristics of nasal consonants, spectral characteristics of selected vowels, estimated slope of the excitation spectrum and duration of a selected vowel. Recognition error rates of 1,5 percent were obtained. The features considered by Sambur (2) were vowel formant frequency and bandwidth and glotcal source poles, location of pole frequencies in nasal consonanc, piech contours and timing characteristics. Sambur found that the most important teatures were timing characteristics, pitch and low -order formants. He obtained an error of 3 percent. Li and Wrench ( 3 ) extended Wakita's cechnique by comparing all the vowels in the unknown utterance wich all che vowels in each reference set. They obtained recognition accuracy ranging from 79 percent to 96 percent.

Marke 2 and Davis [4] used a data of approximately $36 \mathrm{hrs}$. of speech, caken trom interviews. The leacures used were pitch, amplitude, and 20 reflection coefficients. Means and standard deviations for all chese parameters vere computed for varying number of volced $t$ rames.

\section{EXPERTMPATS AND TECENTQUE}

2.1 Linear Prediction Metnod

The basic idea behind the linear prediction method is that sample value of speech, $X[n]$ can be approximated as a linear combination of the past $\mathrm{p}$ speech samples. Mathematically, the linear predictor is described by the equation (5):

$$
\begin{aligned}
x[n] & =a_{1} x[n-1]+a_{2} x[n-2]+\ldots \ldots \ldots \ldots \ldots \ldots \ldots+a_{p} x[n-p] \\
& =\sum a_{k} x[n-k] \quad ; k=1,2 \ldots \ldots .
\end{aligned}
$$

where

$\mathrm{x}[\mathrm{n}]$ is the predicted sample at instant $\mathrm{n}$ and $\mathrm{a}_{1}, \mathrm{a}_{2}, \ldots \mathrm{a}_{\mathrm{p}}$ are the predictor coefficients. It is impossible to predict each signal sample exactly and this leads to a prediction error $\mathrm{E}$ (n) at each sample instant.

$E(n)=x(n)-x^{*}[n]$

Where $X^{\prime}[n]$ is the actual speech sample. 
Mansoura Engineering Journal, (MEJ), Vol.20, No.4, December, 1995. E.63

By minimizing the mean squared error between the actual speech samples and the linearly predicted ones, the predictor coefficiencs can be decermined by solving a set of linear equations, A set of predictor coefficients can predict the speech reasonably accurately over stationary portions. In order to macch the time varying properties of speech, a new set of predictor coefficients are calculated every $10-30$ ms [1]. The problem in linear prediction is to determine the $a_{k}$ coefficients so as to minimize the mean square error, E over a specified number of samples.

$\Sigma=\Sigma E^{2}[n]=\Sigma\{x \cdot[n\}-x[n]\}^{2}$

Or $d E / d a_{j}=-2 \sum x[n-j]\left\{x[n]-\sum a_{n} x[n-k]\right\}=0$

So,

$\sum a_{k} \Sigma(n-j) x(n-k]=\Sigma x[n] x[n-j] ; k=j=1,2, \ldots p$.

Two different efficient methods exit for tinding the solution of this system of equations. These are known as the auto correlation and covariance mechods (6). The multipliers of the au coefficients and the right-hand sides of the system of equation (3) can be put in the form of auto-correlation values of the speech signal for specific sample shifts. These are computed by first multiplying the speech signal $X[n]$ by a soft window function of duration $\mathrm{N}$ samples, and the autocorrelation values are calculated from:

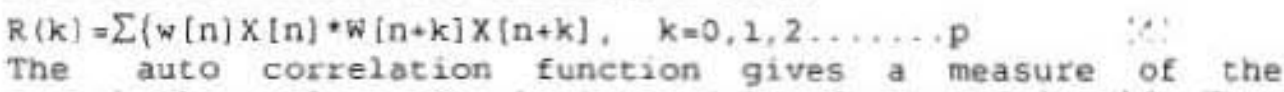
correlacion of a signal with a delayed copy of itselt. These preaictor coefficients will be considered as the feature of the speech samples for each frame.

\subsection{Short Time Energy Punction}

The short time energy function of speech may be computed by splitting the speech signal into frames of $N$ samples and computing the total squared values of the signal samples in each frame. Splitting the signal inco trames can be achieved by multiplying the signal by a suitable window $w(n), n=0,1,2, \ldots N-1$, which is zero for $n$ outside the range $(0, n)$ A simple rectangular vindow of duration $10-20$ ms is suitable for chis purpose. For a window starting at sample m, the short- time energy function $\mathrm{E}_{\mathrm{m}}$ may be written as $[7]$ :

$$
E_{m}=\Sigma(X[n] \cdot w[n-m])^{2}
$$

The energy per frame will be considered as a feature for the speech signa1.

\subsection{The Cepstrum}

The cepscrum of a signal is che fourier transform of che logarithm of its power spectrum. Let $X(\omega)$ denote the specrcum 


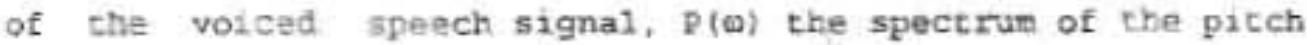
impulses and $H(\varphi)$ the spectrum of the vocal tract which includes the effects of the glottal wave form. The felationship between the magnitude of these three spectra can be expressed simply as follows [B]:

$$
|\mathrm{X}(0)|=|\mathrm{p}| \boldsymbol{\theta})|\cdot| \mathrm{H}(\boldsymbol{\theta}) \mid
$$

Taking the logarithm of this equation gives:

$\log \{x(\omega) \mid\}=\log |D(\omega)|+\log |H(\omega)|$

Thus, in the logarithm of $|x(\omega)|$ the concributions due to $p(\omega)$ and $H(\omega)$ are added. The contribution tron $A(\omega)$, which is essentialiy detemined by the properties of the vocal tragt itself, tends to vary slowly with frequency, while the concribution from $p(\omega)$ terds to vary more rapidly and periodicelly with frequency. These two component should be separable by means of a linear filtering operation. Removing pitch ripple from equation (6) leaves only the vocal tract transfer function. Taking the Fourier cransform of it. ceostrum can be obtained.

2. 4 TIME NOPMAL, TZATION

3y time normalization, is meant the process where time-varying features within the words are brought into line. The classical technique is simply to strecch or compress the unknown word uniformly until it attains the same length as the reference. This process depends for its accuracy on accurate end point identification. Time normalization is now frequently done by a process known as time warping[9]. 'Time warping cechnique is used in this work for time normalization for spoken digits.

\subsection{ENDPOINT ALIGMMNT}

The first step in recognition or adentification is the problem of endpoint alignment (detezmine the location of the spoken word during the recording interval). The algorithm used to determine the endpoinc has been described by Rabinex and Sambur $\mid 10\}$. The end region $1 \mathrm{~s}$ defined as the region from the end of the word to the point at which the energy first exceeds 10. percent of the maximum energy, Bguivalently, an initial region is defined from the beginning of the word to the point at which the energy first exceeds io percent of the maximum. The remaining section is termed the middle region. The midale region is the desired signal.

\subsection{EXPERTMENTS}

Speaker identification becomes more difficult as the size of the speaker population increases, the input template must be compared each with each stored template. Using neural network for.speaker identification may solve this problem.

tive persons have recorded 2250 files.containing Arabic spoken digits. These files are divided into two sets. One set is used for learning the neural network and the other for testing 1 . Improving che accuracy of the performance of the neural 
network can be achieved by using a tree structured network [11] So for speaker identification. chere are nine networks, neural network for every digit. Fig. I shows che block diagram of the hardware implementation of the neural network speaker/speech recognicion system. After endpoint alignment and time normalization, these files are used for preparing the features of each digit. This is done using a PC with speech card and programs in C-language have been written for this propose. The features which have been calculated are energy density per frame in time domain and cepstrum in time for the speech signal. The frame length is taken as $30 \mathrm{~ms}$.

After selection of features, the learning of the neural network is done. in a supervised fashion to compare the performance of each feature. Eor a given collection of input/output pairs of data $\left(x_{1}, c_{2}\right), \ldots,\left(x_{n}, c_{n}\right)$, che parametric learning modifies the parameters of the network minimizing the given performance index $Q$. The general scheme of learning can be concisely expressed as:

\section{$\Delta$ parameter $=-\alpha$ aQ/2parameters}

where $\alpha$ denotes the learning rate. The parameters of network are adjusted following chese increments.

new parameters - actual_parameters + $\Delta$ _parameters

The reader can refer to $[12,13]$ for more details.

3 RESULTS AND DISCUSSION

In patcera recognition, a comparison is made between a test patcern. representing the unknown to be recognized or identified and one or more reference patterns which exist in the pattern library. This comparison takes time depending on the contents of the library. A neural network learns the feacures of the patcerns. So, it takes a very short time for recognition or identification.

The normalized features are applied as inputs to the neural network. For the energy feature, the frame length is taken as 30 ms. After time normalization the longest ward has 40 frames, so the neural network has 40 inputs. 7 ig 2 shows samples of energy-teatures of the different spoken arabic digits by the same speaker. The energy-teacures of digit 'wahed' for different speakers is given in Fig. 3. Any speech contains information about che word being spoken and about the identity of the speaker. In speech recognition we wish to select the first type of features and reduce the effect of the second. The information about che word being spoken can not be isolated from the information about the identity of the speaker. The neural network used for speech recognition has 40 inputs with 4 outputs and one hidden layer with 20 nodes. After learning the retwork and adjusting the neural network parameters, a recognition accuracy of 928 is obtained. A neural network with the same inputs and 3 outputs and one hidden layer with 20 nodes is used to idencify the speaker. 


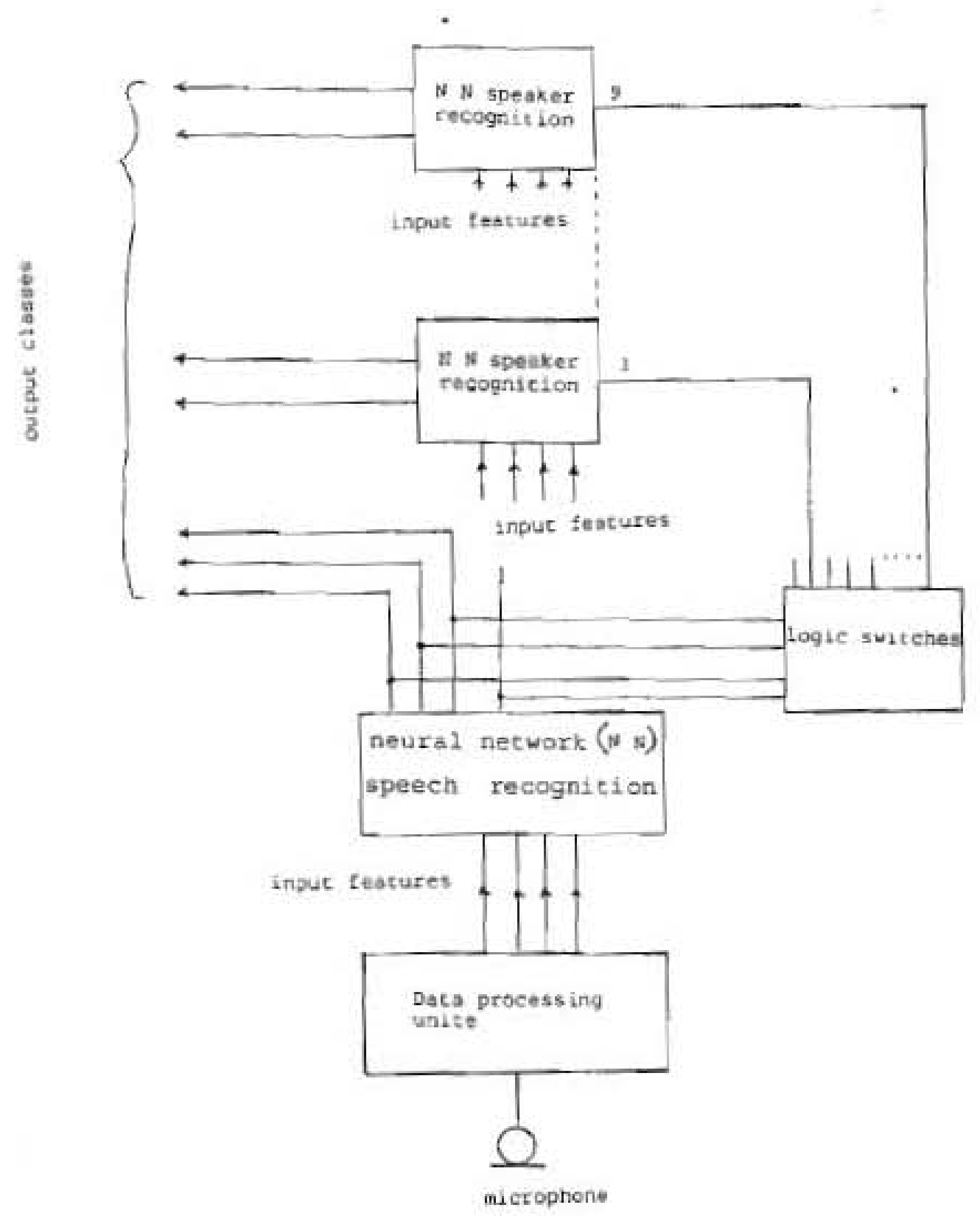

Fig. I The hardware implementation of the neural network speaker - speech recognition system 
Mansoura Engineering Journa1. (MEJ), Vol.20, No.4, December, 1995. E.67
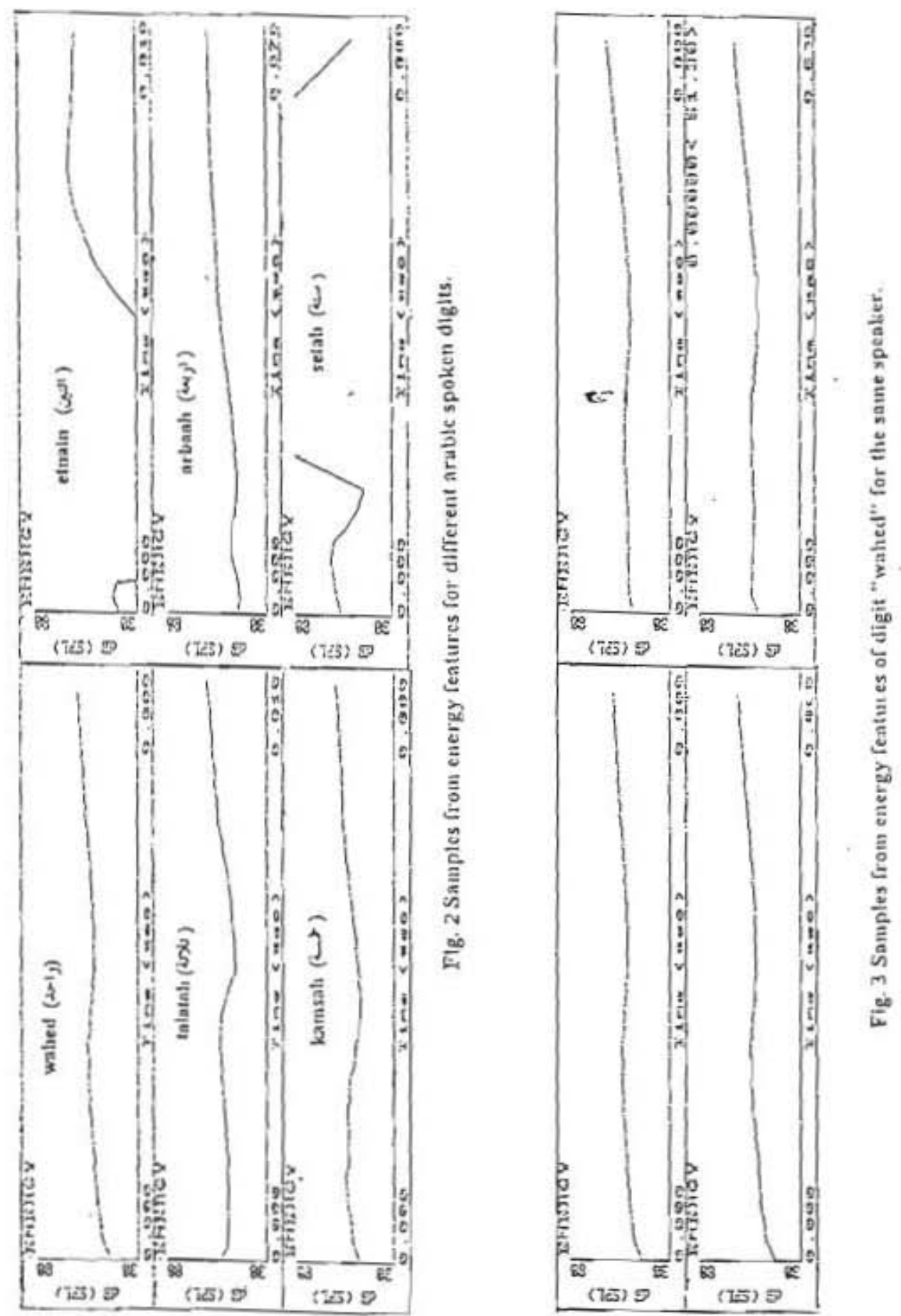

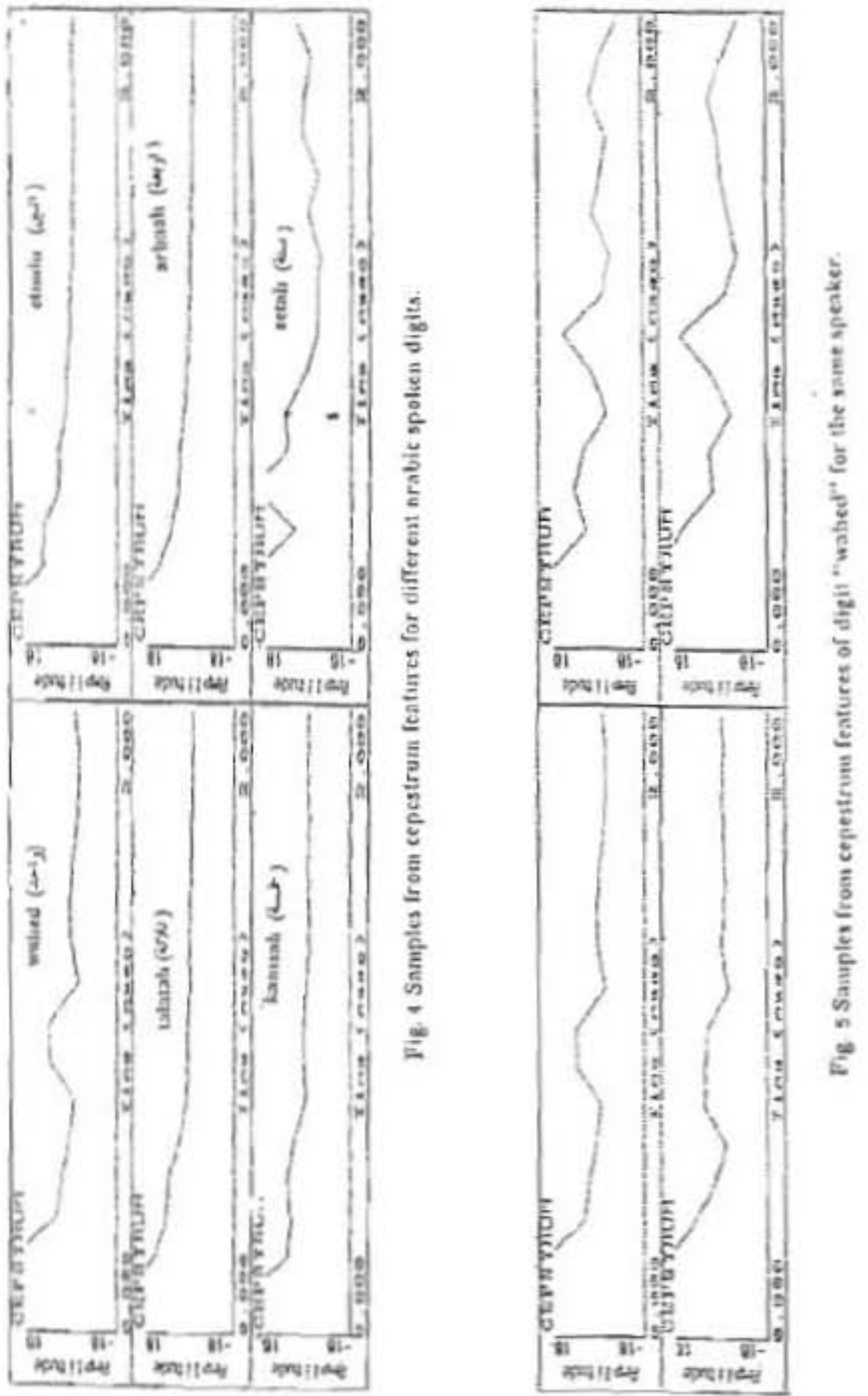
This network may be connected to the recognition necwork through an activated logic swicch. The first network recognizes the digit while the second identifies the speaker. Tables 1 and 2 sumarize the learning results. From these results it is recommended that energy per frame may be used for speech recognition. Also, the response of the vocal cord can be expressed by cepstrum analysis which is considered as an efficient feature. The cepstrum coefficients are obtained in 12 frame. So the neural network for speech recognition has 12 inputs with 4 outputs and one hidden layer with 16 nodes while for speaker identifier a neural network with 12 inputs. 3 outputs, and one hidden layer with 16 nodes. is used. The accuracy of the feature using the cepstrum algorithm is found to be $94 \%$ and $96 \%$ for recognition and identification, respectively.

$\cdot$

Table 1 Test results using energy features for speech recognition

\begin{tabular}{ccc}
\hline Digit & No. of errors & No. of tested files \\
1 & 3 & 10 \\
2 & 0 & 10 \\
3 & 0 & 10 \\
4 & 0 & 10 \\
5 & 0 & 10 \\
6 & 0 & 10 \\
7 & 0 & 10 \\
8 & 4 & 10 \\
9 & 0 & 10 \\
\hline
\end{tabular}

It is clear that the feature of the cepstrum analysis is more accunace than that of energy per frame especially for speaker identification. Tables 3 and 4 summarize the testing results of cepstrum feacures for speech recognition and speaker identification. Figs. 4 and 5 show samples from the cepstrum features of different spoken arabic digits of the same speaker and the cepstrum of digit 'wahad' of different speakers

Table 2 Test results using energy feacures for speaker identification

\begin{tabular}{lcc}
\hline Speaker & No. of errors & No. of tested files \\
speaker1 & 1 & 5 \\
speaker2 & 3 & 5 \\
speaker3 & 2 & 5 \\
speaker4 & 3 & 5 \\
speaker5 & 0 & 5
\end{tabular}


Table I Tedt resulty using cepskram teacures tor specch recognition

\begin{tabular}{lcc}
\hline Digit & No of erzora & No of teated filas \\
1 & 1 & 20 \\
2 & 0 & 19 \\
3 & 0 & 17 \\
4 & 3 & 14 \\
5 & 1 & 21 \\
6 & 1 & 21 \\
7 & 3 & 22 \\
8 & 0 & 20 \\
9 & 0 & 21 \\
\hline
\end{tabular}

Table 4 Test results using cepstrum teatures for speaker identification

\begin{tabular}{lll}
\hline ipeaker & No of errors & No of rasted \\
files & 1 & 5 \\
speaker1 & 0 & 5 \\
speaker2 & 0 & 5 \\
speaker3 & 0 & 5 \\
speakera & 0 & 5 \\
speaker5 & & \\
\hline
\end{tabular}

Mostafa [14] used the LPC coefficients only as a feacure to investigate the speaker ident:fier system using neural network His results show that the accuracy of LPC was found to be 958 .

\section{Cosclusion}

The objective of this article is to investigate the speech feacures and a comparison between these features is given. It is found that the cepscrum is a powerful feature selection technique especially for speaker identification. A neural network speaker -speech recognition system is given with complete analysis for the arabic spoken digics

\section{REREREDCES}

1. Wolf.J J.. "Efticient Acoustic Paraneters For Speaker Recognition", JhSA, Fol.51, June, 1972.

2.Sambur, M. R.. "Selection of Acoustic Features Por Speaker Identification", IEEE-Trans.. vol.ASSP-23, no.2, Apri1. 1975 .

3.Li, R. and Wrench, B. H., "Mn approach to cext-independent speaker recognition with short utterances"ICASSP-63, 1983. 
- 4.Markel, J. D. and David, S. B., "Text-1ndependent spazker recognition from a large linguistically unconstrained timespaced datz base*, IEEE Trans.,vol. ASSP-27.No.1, FEB.T, 1979.

5. Parsons, T, W.. "Voice and Speech Processing*, McGraw-Hill Book Company, New York, 1987.

6. Owens, F. J.., "Signal Processing of Speech", Macmillan Preas LTD, Hong Kong 1993.

7. Schafer, R, W and Rabiner L. "Parametric Representation of Speech", IEEE Syaposıum in 1974, ACADEMIC PRES5, New York 1975.

8. Furui. 5.. "Cepstrum Analysis Por Automacic Speaker Verification", IEEB Trans.,vol. ASSP, No,2, April 1981.

9.Sakoe, H. and Chiba, S. "Dynamic Programing Alogrich Optimizat: on for Spoken word Recignition", IBEB Transaction on Acoustic, Speech and Signal Processing, wol. ASSP-26, Feb. 1975

10. Rabiner $\mathrm{L}$. and Sambur M., "An algorith for Determining the Bndpoints of Isolated Utcerances", The Bell System Tech. Journal, vol.64. No.2, Feb.1975.

11. Rashwan, M. A. A. and others, -Improving Classification Using a Tree Structured Neural Network", Journal of Intelligent and Fuzzy Systems, Vol.2, 1994.

12. Pao, Y., "Adaptive Patern Recognition and Neural Networks", Addison-Wesley Publishing Conpany, INC, New York, 1989.

13. Nguyen, $D$ and Widrow, B... "Neural Networks for SelcLearning Control System", tREB Contr. Syst. Mag., Vol.10, No. 3, April 1990.

14. Mostafa W., "Application of Neural Network on Speaker Recognition", M.Sc, thesis, Faculty of Eng Cairo univ, ,1995 